\title{
Long-term total sleep deprivation reduces thalamic gray matter volume in healthy men
}

Article in Neuroreport · December 2013

DOI: 10.1097/WNR.0000000000000091 · Source: PubMed

CITATIONS

3

5 authors, including:

Chunlei Liu

Beijing Normal University

6 PUBLICATIONS 15 CITATIONS

SEE PROFILE

\section{Renlai Zhou}

Nanjing University

77 PUBLICATIONS 263 CITATIONS

SEE PROFILE
READS
Xiang-Zhen Kong

Max Planck Institute for Psycholinguistics

23 PUBLICATIONS 68 CITATIONS

SEE PROFILE

\section{Bin Wu}

166 PUBLICATIONS 1,113 CITATIONS

SEE PROFILE 


\section{Long-term total sleep deprivation reduces thalamic gray matter volume in healthy men Chunlei Liu ${ }^{a, *}$, Xiang-zhen Kong ${ }^{b, *}$, Xueyong Liu ${ }^{d}$, Renlai Zhou ${ }^{a, b, c}$ and Bin Wu}

\begin{abstract}
Sleep loss can alter extrinsic, task-related functional MRI signals involved in attention, memory, and executive function. However, the effects of sleep loss on brain structure have not been well characterized. Recent studies with patients with sleep disorders and animal models have demonstrated reduction of regional brain structure in the hippocampus and thalamus. In this study, using T1weighted MRI, we examined the change of regional gray matter volume in healthy adults after long-term total sleep deprivation $(\sim 72 \mathrm{~h})$. Regional volume changes were explored using voxel-based morphometry with a paired two-sample $t$-test. The results revealed significant loss of gray matter volume in the thalamus but not in the hippocampus. No overall decrease in whole brain gray matter volume was noted after sleep deprivation. As expected, sleep deprivation significantly reduced visual vigilance as assessed by the continuous performance test, and this decrease was correlated significantly with reduced regional gray matter volume in thalamic regions. This study provides the first evidence for sleep loss-related changes
\end{abstract}

\section{Introduction}

Previous studies have demonstrated the effect of sleep deprivation (SD) on psychological measures and neural systems [1-6]. It has been demonstrated that sleep loss can reduce performance on a broad variety of tasks and affects brain functional activity $[7,8]$. Nevertheless, no previous study has examined the effects of sleep loss on gray matter (GM) structures in healthy adults. Considering sleep loss decreases a wide range of cognitive functions, the association of reduced performance and GM structure changes is a matter of interest. To date, no human studies have provided direct evidence that sleep loss affects the structure of the brain. Case-control studies with patients with sleep problems (e.g. insomnia, obstructive sleep apnea, depression, and sleepiness during the day) have attempted to test the hypothesis that chronically disturbed sleep is associated with changes in hippocampal and thalamus brain structure [9-11]. These related studies, have provided some hints about the effects of SD on human brain structure, but these models are not representative of sleep loss in healthy human adults.

In this study, we investigated this issue using voxel-based morphometry (VBM) and long-duration $(\sim 72 \mathrm{~h})$ SD. Before and after the intervention, each individual participated in MRI experiments. We hypothesized that there are changes induced by SD in the regional GM structures of healthy young adults, such as volume reductions in the hippocampus and thalamus. in gray matter in the healthy adult brain. NeuroReport 25:320-323 @ 2014 Wolters Kluwer Health | Lippincott Williams \& Wilkins.

NeuroReport 2014, 25:320-323

Keywords: brain structure, gray matter volume, sleep deprivation, thalamus, voxel-based morphometry

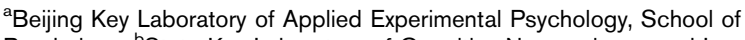
Psychology, ' $S$ tate Key Laboratory of Cognitive Neuroscience and Learning, ${ }^{\mathrm{C}}$ Center for Collaboration and Innovation in Brain and Learning Sciences, Beijing Normal University and National Key Laboratory of Human Factors Engineering, China Astronaut Research and Training Center, Beijing, China

Correspondence to Renlai Zhou, PhD, Beijing Key Laboratory of Applied Experimental Psychology, School of Psychology, Beijing Normal University, Beijing 100875, China

Tel/fax: + 861058802021 ; e-mail: rlzhou@bnu.edu.cn

or

Bin $\mathrm{Wu}, \mathrm{PhD}$, National Key Laboratory of Human Factors Engineering, China Astronaut Research and Training Center, Beijing 100094, China

Tel/fax: +8610 68117398; e-mail: binwuacc@gmail.com

*Chunlei Liu and Xiang-zhen Kong contributed equally to the writing of this article.

Received 2 October 2013 accepted 8 November 2013

\section{Methods}

\section{Participants}

Twelve healthy college students (aged between 20 and 32 years, mean $=24.83, \mathrm{SD}=2.88$; right-handed; all males) were recruited from Beijing Normal University (BNU) (Beijing, China). All participants received a psychiatric interview, physical examination, and routine laboratory screening, they were free of physical and mental disorders, and were all right-handed. The experimental protocol was approved by the Institutional Review Board of BNU. In accordance with the Declaration of Helsinki (1991), written informed consent was obtained from each participant before the investigation.

\section{Sleep deprivation}

The SD experiment was conducted in the SD laboratory at the Astronaut Center of China. Participants were studied with MRI twice, at the same time of day (8:00-12:00 a.m.): once after a normal night of sleep (mean duration of sleep, $7 \pm 1 \mathrm{~h}$ ) and once after total sleep deprivation $(72 \pm 0.8 \mathrm{~h})$. Before the SD, all participants experienced a normal night of sleep in bed, which was recorded by polysomnography. During the 72-h SD period, participants were required to stay awake at all times. Three research assistants remained with them throughout the day and night to ensure that they did not fall asleep for the SD. If there were any signs of a 
participant falling asleep, they were awakened by an alarm clock immediately. All participants experienced roughly the same amount of sleep loss.

On the day before SD, a T1-weighted image was acquired from each participant, after which the SD was initiated. After the SD, all participants, except one, participated in the post-test of structural MRI scanning. During the break in the scanning, the participants did the continuous performance test, a visual vigilance task [12], which they had practiced previously. This involves a button press response to stimuli occurring at 2-s intervals, ensuring that subjects remain awake during MRI scanning.

\section{MRI data acquisition}

MRI were acquired on a 3-T Siemens Trio MRI scanner (Siemens, Erlangen, Germany) at the Beijing Normal University Imaging Center for Brain Research (Beijing, China). A 3D magnetization-prepared rapid gradientecho (MP-RAGE) MRI sequence $(\mathrm{TR} / \mathrm{TE} / \mathrm{TI}=2530 /$ $3.39 / 1100 \mathrm{~ms}$, flip angle $=7^{\circ}$, voxel size $=1 \times 1 \times 1.33$ $\mathrm{mm}, 128$ slices) was used to obtain the T1-weighted image of the entire brain, and data were acquired twice before and after the SD period for each participant.

\section{MRI data preprocessing}

First, the quality of the T1-weighted MRI was assessed by visual examination. Second, these images were segmented for GM using the segmentation tools in VBM8 from Statistical Parametric Mapping 8 (SPM8, http://wrwe.fil.ion.ucl.ac.uk/spm). The GM images for each participant were then normalized to a study-specific template in MNI152 space using diffeomorphic anatomical registration through exponential lie algebra [13]. To ensure that the total amount of GM was conserved after spatial transformation, we modulated the transformed images by the Jacobian determinants of the deformation field. The modulated GM images were then smoothed with a Gaussian kernel (full width at half maximum $=8$ $\mathrm{mm}$ ) before being used for further analyses.

\section{Region of interest analysis}

We obtained masks for the bilateral hippocampus and thalamus from a widely used human brain probability template, the Harvard-Oxford subcortical structural atlas. We chose to use the common $25 \%$ threshold subtemplate. To rule out the possibility that any potential effects observed with the $25 \%$ atlas were driven by peripheric region inclusion, analyses were also conducted with the $50 \%$ atlas. The results of this additional analysis were highly convergent with those for the $25 \%$ atlas (data not shown). In each analysis, a paired two-sample $t$-test was performed on the mean GM volume of each region of interest (ROI) to determine whether they displayed a SD-caused reduction. The mean GM volume within these regions was calculated with an in-house Pythoncoded tool.

\section{Whole-brain analyses}

For the areas outside the ROIs, we conducted a paired $t$-test on each voxel across the whole brain before and after the SD. However, we did not find regions that displayed significant reduction because of sleep loss with appropriate corrections for false-positive error at a threshold of $P$ less than 0.05 . To search for other possible candidate regions for future studies, we also performed an analysis with a slightly more lenient criterion $(P<0.001$, uncorrected, cluster size $>40 \mathrm{~mm}^{3}$ ).

\section{Results \\ Task performance}

As expected, vigilance performance significantly declined after SD $\left(d^{\prime}=1.82 \pm 1.01\right)$ compared with normal sleep $\left[d^{\prime}=2.56 \pm 0.95, t(10)=2.87, P<0.01\right] \quad\left(d^{\prime}\right.$ represents that portion of the signal-noise distribution attributable to veridical perception of the signal according to the signal detection theory, and thus $d^{\prime}$ provides a means for assessing an individual's discriminative power).

\section{Gray matter volume}

To test the hypothesis that SD reduces the regional GM volume in the thalamus and hippocampus, we recorded structural MRI scans from 11 healthy young adults (male) before and after a long-duration SD. We then used VBM analysis to investigate the change of regional GM volume in these regions after the SD period. Among the four candidate regions, we found that the regional bilateral thalamus GM volume was significantly reduced (survived Bonferroni correction for multiple testing) after SD [left: $t(10)=3.832, P=0.006$; right: $t(10)=3.924, P=0.006$ ] However, we did not observe any significant hippocampal changes [left: $t(10)<1$; right: $t(10)<1$ ]. The two bilateral brain subcortical regions and changes of regional GM volume averaged across the bilateral ROIs are shown in Fig. 1.

Outside these ROIs reflecting our prior hypotheses, we also conducted a whole-brain analysis to reveal any additional brain structures that displayed reduction because of sleep loss. However, no regions displayed a reduction that survived correction for multiple comparisons across the whole brain $\left(P_{\mathrm{FDR}}>0.05\right)$. With a more lenient statistical criterion $(P<0.001$ uncorrected and cluster size $>40 \mathrm{~mm}^{3}$ ), we found clusters in which GM volume was significantly reduced (Table 1 ) after SD in the left middle temporal gyrus $(Z=3.91, P<0.0005)$, right inferior temporal gyrus $(Z=4.49, P<0.0005)$, left postcentral gyrus $(Z=3.81, P<0.0005)$, right lateral occipital cortex $(Z=3.91, P<0.0005)$, and bilateral cerebellum (right: $Z=3.9, P<0.0005$; left: $Z=4.01$, $P<0.0005 ; Z=3.68, P<0.0005)$. No regions displayed an increase after $\mathrm{SD}$, as we had expected. In addition, the reduction in total $\mathrm{GM}$ volume after $\mathrm{SD}$ was not significant $[t(10)=1.61, P=0.15]$. Thus, our data 
Fig. 1
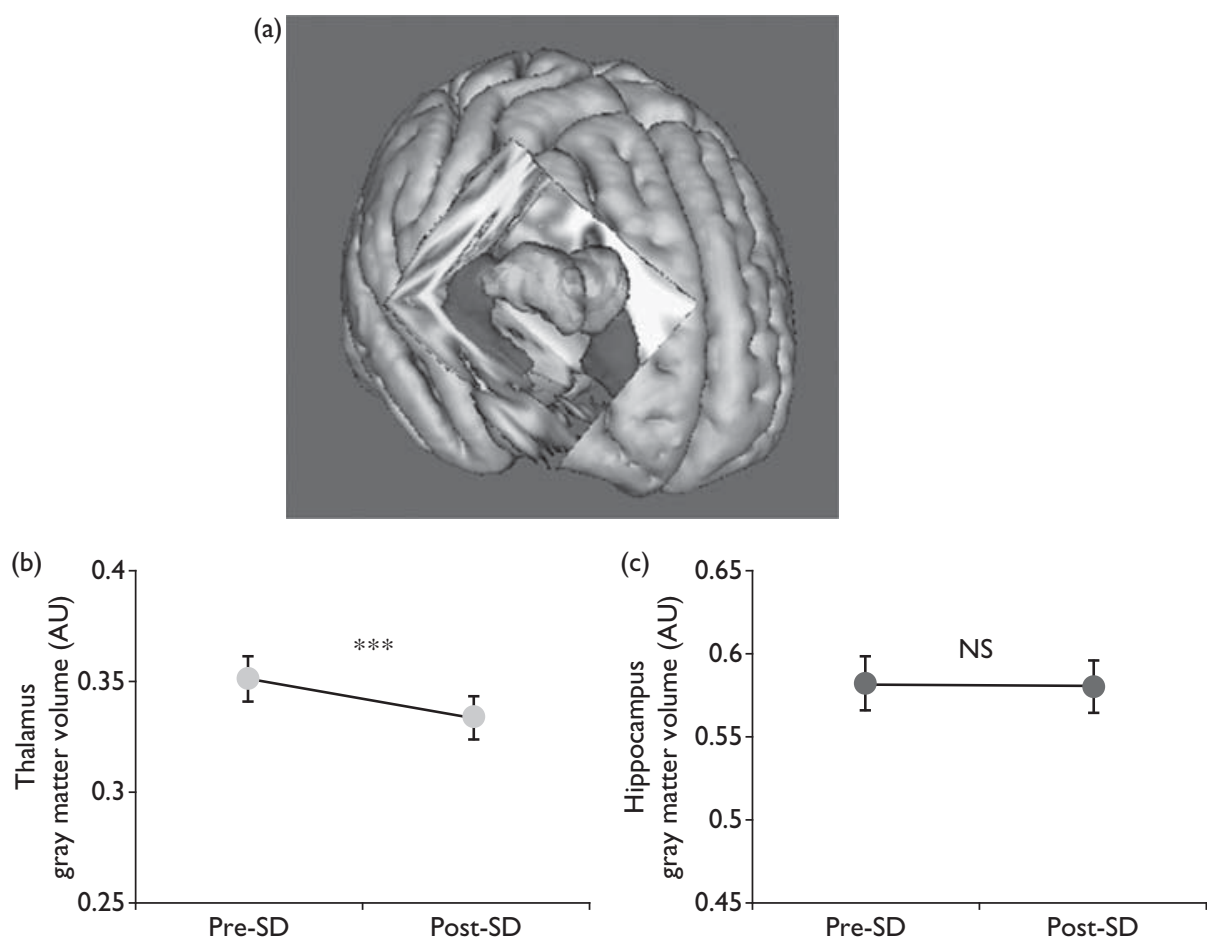

Reduction of regional gray matter (GM) volume after sleep deprivation (SD). (a) Two subcortical brain structures are shown in different colors. Green indicates the bilateral thalamus and purple indicates the bilateral hippocampus. (b) The region of interest (ROI) in the thalamus (left) displayed significant reduction of GM volume after SD. The changes (right) in regional GM volume averaged across the bilateral ROls are shown. (c) The region of interest (ROI) in the hippocampus (left) displayed significant reduction of GM volume after SD. The changes (right) in regional GM volume averaged across the bilateral ROls are shown. $* * * P<0.001 ; \mathrm{NS}, P>0.05$. Error bars represent the SEM.

Table 1 Brain structures that displayed reduction after sleep deprivation in the whole-brain analysis

\begin{tabular}{|c|c|c|c|c|c|c|c|}
\hline \multirow{2}{*}{$\begin{array}{l}\text { Brain region } \\
\text { Thalamus }\end{array}$} & \multirow{2}{*}{$\frac{\mathrm{L} / \mathrm{R}}{\mathrm{R}}$} & \multicolumn{3}{|c|}{ Maxima of cluster (mm) } & \multirow{2}{*}{$\frac{Z}{3.94}$} & \multirow{2}{*}{$\begin{array}{c}P \\
0.000041\end{array}$} & \multirow{2}{*}{$\frac{\text { Cluster size (voxels) }}{110}$} \\
\hline & & 16 & -30 & 12 & & & \\
\hline \multirow[t]{3}{*}{ Cerebellum } & $\mathrm{R}$ & 36 & -60 & -56 & 3.9 & 0.000048 & 106 \\
\hline & $\mathrm{L}$ & -16 & -64 & -50 & 4.01 & 0.00003 & 50 \\
\hline & $\mathrm{L}$ & -30 & -90 & -36 & 3.68 & 0.00012 & 41 \\
\hline Postcentral gyrus & $\mathrm{L}$ & -54 & -4 & 36 & 3.81 & 0.00007 & 55 \\
\hline Inferior temporal gyrus & $\mathrm{R}$ & 54 & -50 & -16 & 4.49 & 0.0000035 & 49 \\
\hline Middle temporal gyrus & $\mathrm{L}$ & -58 & -56 & 4 & 3.91 & 0.000045 & 43 \\
\hline Lateral occipital cortex & $\mathrm{R}$ & 52 & -62 & 16 & 3.91 & 0.000045 & 41 \\
\hline
\end{tabular}

L, left; R, right.

indicated regional bilateral thalamus specificity for the loss of GM after SD.

\section{Regional gray matter volume correlation with task}

In this study, the greater the decrease in attention scores from rest to SD, the greater the reduction in GM volume (data combined for right and left side). These correlations were positive in the thalamus $(r=0.83, P<0.01)$. No lateral cortical lobe correlations reached significance.

\section{Discussion}

The present study revealed the effect of long-term total SD on GM volume. Consistent with our hypothesis, sleep loss actually reduced regional GM volume in the human brain. In particular, we observed reduced regional GM volume in the bilateral thalamus.

Some studies reveal that experiencing sleep loss can cause a reduction of regional GM volume, for example, in human patients with primary insomnia [9]. With healthy younger adults and within-subject design, we first observed a significant reduction in regional GM in the bilateral thalamus, which is considered to be connected to consciousness as it functions as the gatekeeper for all sensory information in the human brain $[14,15]$. As the thalamus connects different areas of the brain to each other, it is involved in the relaying of sensory signals to 
the cerebral cortex; reception of auditory, physical, and visual signals; motor control; and regulation of cognitive and emotional processing [16]. Thus, if the human thalamus is damaged physically or neurologically (e.g. from stroke, accident, medication, or genetics), such damage can lead to problems with sleeping, pain perception, memory issues, and sensory impairment, among other areas. For example, previous studies observed increased thalamic responses during SD for working memory [17,18] and attention tasks [19,20], which might reflect a 'compensatory' response caused by regional GM reduction in the thalamus. Thus, the observed structural reduction in the thalamus caused by SD may underlie the observed decreased cognitive and emotional performance and reflect the nonspecific mechanisms that support cortical function and maintain steady performance of the thalamus. In addition, the reduction of regional brain structure volume may provide us with new insight into the clinical implications of sleep loss.

In contrast to previous studies, we did not observe reduced regional GM in the hippocampus. Although several studies in rats and insomnia patients have reported reduced hippocampal volumes, the mechanism underlying this reduction is not clear. For example, in studies with insomnia patients, findings may be clouded by other comorbidities. Unlike those studies using patients or nonhuman mammals as participants, we recruited healthy younger adults and conducted SD for an entire $72 \mathrm{~h}$. In fact, reduced regional GM in the hippocampus apparently does not occur in young adults after acute $72-\mathrm{h}$ $\mathrm{SD}$, which contrasts with the reduction in hippocampal volume seen in patients with insomnia. Additional research on the changes in the hippocampus after SD is needed.

\section{Conclusion}

We found that long-term SD reduces thalamic regional GM volume in the human brain. To the best of our knowledge, this is the first report on the effects of total $\mathrm{SD}$ on regional brain structure during wakefulness in humans, using VBM. Although these results should be regarded as preliminary and require replication and extension in future studies, they do suggest that the changes in brain structure during and after SD can inform our understanding of the effects of SD on cognitive and emotional performance from the perspective of brain structure.

\section{Acknowledgements}

This work was funded by the Main Test Technique Research Program of China (2011CB711000) and the
'973' project (2011CB711001). The authors express their gratitude for support of this project.

\section{Conflicts of interest}

There are no conflicts of interest.

\section{References}

1 Waters F, Bucks RS. Neuropsychological effects of sleep loss: implication for neuropsychologists. J Int Neuropsychol Soc 2011; 17:1-16.

2 McCoy JG, Strecker RE. The cognitive cost of sleep lost. Neurobiol Learn Mem 2011; 96:564-582.

3 WDS Killgore. Effects of sleep deprivation on cognition. In: Gerard AK, PAvD Hans, editors. Progress in brain research. Amsterdam: Elsevier; 2010. pp. 105-129.

4 Basner M, Rao H, Goel N, Dinges DF. Sleep deprivation and neurobehavioral dynamics. Curr Opin Neurobiol 2013; 23:854-863.

5 Boonstra TW, Stins JF, Daffertshofer A, Beek PJ. Effects of sleep deprivation on neural functioning: an integrative review. Cell Mol Life Sci 2007; 64: 934-946.

6 Goel N, Rao H, Durmer JS, Dinges DF. Neurocognitive consequences of sleep deprivation. Semin Neurol 2009; 29:320-339.

7 Piantoni G, Cheung BL, Van Veen BD, Romeijn N, Riedner BA, Tononi G, et al. Disrupted directed connectivity along the cingulate cortex determines vigilance after sleep deprivation. Neuroimage 2013; 79:213-222.

8 De Havas JA, Parimal S, Soon CS, Chee MWL. Sleep deprivation reduces default mode network connectivity and anti-correlation during rest and task performance. Neuroimage 2012; 59:1745-1751.

9 Riemann D, Voderholzer U, Spiegelhalder K, Hornyak M, Buysse DJ, Nissen $\mathrm{C}$, et al. Chronic insomnia and MRI-measured hippocampal volumes: a pilot study. Sleep 2007; 30:955

10 Guzman-Marin R, Suntsova N, Methippara M, Greiffenstein R, Szymusiak R, McGinty D. Sleep deprivation suppresses neurogenesis in the adult hippocampus of rats. Eur J Neurosci 2005; 22:2111-2116.

11 Volkow ND, Wang GJ, Telang F, Fowler JS, Logan J, Wong C, et al. Sleep deprivation decreases binding of $\left[{ }^{11} \mathrm{C}\right]$ raclopride to dopamine D2/D3 receptors in the human brain. J Neurosci 2008; 28:8454-8461.

12 Wu JC, Gillin JC, Buchsbaum MS, Hershey T, Hazlett E, Sicotte N, et al. The effect of sleep deprivation on cerebral glucose metabolic rate in normal humans assessed with positron emission tomography. Sleep 1991; 14:155-162.

13 Ashburner J. A fast diffeomorphic image registration algorithm. Neuroimage 2007; 38:95-113.

14 Roca J, Fuentes LJ, Marotta A, Lopez-Ramon MF, Castro C, Lupianez J, et al. The effects of sleep deprivation on the attentional functions and vigilance. Acta Psychol (Amst) 2012; 140:164-176.

15 Llinas R, Ribary U, Contreras D, Pedroarena C. The neuronal basis for consciousness. Philos Trans R Soc London [Biol] 1998; 353:1841-1849.

16 Portas CM, Rees G, Howseman A, Josephs O, Turner R, Frith C. A specific role for the thalamus in mediating the interaction of attention and arousal in humans. J Neurosci 1998; 18:8979-8989.

17 Chee MW, Chuah LY, Venkatraman V, Chan WY, Philip P, Dinges DF. Functional imaging of working memory following normal sleep and after 24 and $35 \mathrm{~h}$ of sleep deprivation: correlations of fronto-parietal activation with performance. Neuroimage 2006; 31:419-428.

18 Vandewalle G, Archer SN, Wuillaume C, Balteau E, Degueldre C, Luxen A, et al. Functional magnetic resonance imaging-assessed brain responses during an executive task depend on interaction of sleep homeostasis, circadian phase, and PER3 genotype. J Neurosci 2009; 29:7948-7956.

19 Chee MWL, Goh CSF, Namburi P, Parimal S, Seidl KN, Kastner S. Effects of sleep deprivation on cortical activation during directed attention in the absence and presence of visual stimuli. Neuroimage 2011; 58:595-604.

20 Chee MW, Tan JC. Lapsing when sleep deprived: neural activation characteristics of resistant and vulnerable individuals. Neuroimage 2010; 51:835-843. 